\title{
Metabolic Disturbances Independent of Body Mass in Patients with Schizophrenia Taking Atypical Antipsychotics
}

\author{
Shi Hyun Kang ${ }^{\varpi}$ and Jong II Lee \\ Department of Psychiatry, Seoul National Hospital, Seoul, Republic of Korea
}

\begin{abstract}
Objective Atypical antipsychotic (AAP) treatment is associated with weight gain and metabolic disturbances such as dyslipidemia and dysglycemia. The metabolic disturbances are usually considered to develop secondary to weight gain. We performed the comparison of metabolic disturbances of three AAP group with different risk of metabolic side effect after adjusting for body mass to investigate whether any metabolic disturbances develop independently from body mass index (BMI).

Methods This cross-sectional study included 174 subjects with schizophrenia who were on 1) monotherapy with clozapine (CL), olanzapine (OL), or quetiapine (QT) (n=61), 2) monotherapy with risperidone (RSP) (n=89), or 3) monotherapy with aripiprizole (ARP), or ziprasidone (ZPS) ( $\mathrm{n}=24)$ more than 1 year. Association between the prevalence of metabolic disturbances and groups were analysed using logistic regression after adjusting confounding variables including BMI. Analysese of covariance were used to compare the AAP groups in terms of the levels of metabolic parameters.

Results There were significant differences among groups in terms of the prevalence of hypertriglyceridemia ( $\mathrm{p}=0.015$ ), low HDL-cholesterol $(\mathrm{p}=0.017)$, and hyperglycemia $(\mathrm{p}=0.022)$ after adjusting for BMI. Triglyceride level $(\mathrm{p}=0.014)$ and the ratio of triglyceride to HDLcholesterol ( $\mathrm{p}=0.004)$ were significantly different among groups after adjusting for BMI.

Conclusion In conclusion, metabolic disturbances are significantly different in AAP groups even after adjusting BMI. AAPs may have direct effect on metabolic parameters. Blood lipid and glucose levels should be monitored regularly regardless of whether patients tend to gain weight.

Psychiatry Investig 2015;12(2):242-248
\end{abstract}

Key Words Schizophrenia, Dyslipidemia, Hyperglycemia, Atypical, Antipsychotics, Metabolic.

\section{INTRODUCTION}

Since their introduction in 1990, atypical antipsychotics (AAPs) have become the most common treatment for patients with a variety of psychotic disorders. ${ }^{1}$ However, compelling evidence indicates that the use of AAPs is related to potentially serious adverse metabolic effects, including obesity, dyslipidemia, hyperglycemia, and type 2 diabetes mellitus., ${ }^{2,3}$ These metabolic disturbances may lead to an increase in the risk of cardiovascular diseases and premature mortality. ${ }^{4}$

A growing body of research has compared the metabolic side effects of different AAPs, and the findings indicate that

Received: November 13, 2013 Revised: March 29, 2014

Accepted: May 26, 2014 Available online: March 18, 2015

$\triangle$ Correspondence: Shi Hyun Kang, MD, PhD

Department of Psychiatry, Seoul National Hospital, 398 Neungdong-ro, Gwangingu, Seoul 143-711, Republic of Korea

Tel: +82-2-2204-0326, Fax: +82-2-2204-0394, E-mail: drshe@hanmail.net

(c) This is an Open Access article distributed under the terms of the Creative Commons Attribution Non-Commercial License (http://creativecommons.org/licenses/bync/3.0) which permits unrestricted non-commercial use, distribution, and reproduction in any medium, provided the original work is properly cited. different AAPs are associated with different levels of risk for metabolic disturbances. ${ }^{5-7}$ However, the pathophysiological mechanisms underlying metabolic side effects are not fully understood. AAP-induced weight gain has been the primary focus of attention because obesity is an easily detectable side effect, and researchers have assumed that the initiating pathophysiology is weight gain. Obesity is known to predispose individuals to develop dyslipidemia and glucose intolerance Thus, it is conceivable that AAP-associated metabolic disturbances are associated with weight gain, and this has been supported by several reports. ${ }^{1}$ Nevertheless, considerable evidence suggests that AAPs can cause metabolic disturbances in a manner that cannot be explained by weight gain alone. A cross-sectional naturalistic study of 242 individuals with severe mental disorders revealed that, despite similar body mass indices (BMIs), hypertriglyceridemia was significantly more prevalent in clozapine- or olanzapine treated than in unmedicated subjects. ${ }^{2}$ Moreover, several studies of AAPs have found no strong association between the severity of the observed hypertriglyceridemia and weight gain. ${ }^{3,46}$ Henderson et al. ${ }^{8}$ reported that clozap- 
ine- and olanzapine treated subjects displayed significant insulin resistance and impaired glucose effectiveness compared with BMI-matched risperidone-treated subjects. These findings may suggest that AAPs directly affect metabolic disturbances, rather than simply increase the effects of known risk factors such as obesity. Therefore, the association between AAP-induced metabolic disturbances and obesity needs to be further investigated. A precise understanding of the mechanism underlying this association should enable the development of effective measures to reduce the metabolic risk for patients taking AAPs.

In the present study, we compared the metabolic disturbances of three groups treated with different AAPs, which are known to differ in terms of the risk of weight gain, after adjusting for BMI. The primary objective of this study is to investigate whether any metabolic disturbances differ independently from body mass in patients with schizophrenia taking three AAP group with different risk of metabolic side effect.

\section{METHODS}

\section{Subjects}

The original research by Kang et al. ${ }^{9}$ focused on the prevalence of metabolic syndrome (MetS) among patients with schizophrenia or schizoaffective disorders. In brief, they conducted a study at the outpatient and inpatient departments of Seoul National Hospital in Korea. Their sample included 957 patients diagnosed with schizophrenia or schizoaffective disorder who were 18-65 years of age and who had been taking the same antipsychotics for more than one year. The Diagnostic and Statistical Manual of Mental Disorders, 4th edition, Text Revision (DSM-IV-TR) diagnosis was established by chart review. All subjects provided written informed consent of the study procedures, which were approved by the institutional review board at Seoul National Hospital.

The present study is a post-hoc analysis of the Dr. Kang's study. ${ }^{9}$ Of the 957 subjects in the original study, only those who have been receiving monotherapy with an AAP [clozapine (CL), olanzapine (OL), quetiapine (QT), risperidone (RSP), aripiprizole (ARP), or ziprasidone (ZPS)] were included in the present study. The following exclusion criteria were also applied: 1) treatment with any mood stabilizer (lithium, valproate, carbamazepine, lamotrigine, topiramate); 2) treatment with several antidepressants known to induce weight gain (mirtazapine, tricyclic antidepressants); and 3) use of cigarettes (because smoking may influence weight and metabolic disturbances).

This sample was divided into three AAP groups according to the risk of metabolic side effects: 1) CL/OL/QT, patients treated with CL, OL, or QT; 2) RSP, patients treated with RSP; and 3) ARP/ZPS, patients treated with either ARP or ZPS. The dibenzodiazepine-derived antipsychotics are known to induce severe metabolic adverse effects. ${ }^{7}$ There are several reports showing differences of metabolic side effects between RSP and ARP/ZPS. ${ }^{10,11}$

\section{Procedures}

Data were collected on age, sex, duration of illness, current Clinical Global Impression-Severity (CGI-S) score. Data on the dosages and duration of current antipsychotic agent were obtained from medical records. Information on the presence of and current medication regimen for diabetes, hypertension, and dyslipidemia was gathered via patient self-reports.

Fasting blood samples were taken in the morning after an 8 -h overnight fast. Fasting plasma glucose, total cholesterol, triglycerides, and high-density lipoprotein (HDL) cholesterol were measured in a central certified laboratory. Blood pressure (BP) was measured in the sitting position after a 10-min rest period. Body weight, height and waist circumference and were measured and BMI was calculated by weight $(\mathrm{kg}) / \mathrm{height}\left(\mathrm{m}^{2}\right)$.

The present study used the criteria for MetS proposed by the National Cholesterol Education Program (NCEP) adapted Adult Treatment Panel III (ATP IIIA), ${ }^{12}$ which defined this condition as the presence of three or more of the following risk factors: central obesity; hypertriglyceridemia, with fasting plasma triglycerides of $\geq 150 \mathrm{mg} / \mathrm{dL}$; low HDL cholesterol with fasting HDL cholesterol of $<40 \mathrm{mg} / \mathrm{dL}$ in men and $<50 \mathrm{mg} / \mathrm{dL}$ in women; hypertension, with systolic and/or diastolic blood pressure of $\geq 130 / 85 \mathrm{~mm}$ Hg or known treatment for hypertension; and hyperglycemia, with fasting plasma glucose of $\geq 100$ $\mathrm{mg} / \mathrm{dL}$ or known treatment for diabetes. We used the definition of abdominal obesity for Asian populations: $\geq 90 \mathrm{~cm}$ in men and $\geq 80 \mathrm{~cm}$ in women from the Western Pacific regional office of the WHO. ${ }^{13}$ The prevalence rate for obesity was determined using definitions of $\mathrm{BMI} \geq 30 \mathrm{~kg} / \mathrm{m}^{2}$.

\section{Statistical analyses}

Demographic and clinical variables are presented as mean values \pm SDs or as proportions. Chi-square tests were performed for dichotomous variables, and analyses of variance were performed for continuous variables. The post-hoc analyses were performed using Bonferroni method. Associations between the prevalence of MetS, obesity, and treatment groups were analyzed using logistic regression analyses after adjusting for the potential confounding variables of age, sex, duration of current AAP treatment, and haloperidol (HPD)-equivalent dosage of current AAP. Associations between the prevalence of dyslipidemia, hypertension, dysglycemia, and treatment group were analyzed using logistic regression analyses after adjusting for the potential confounding variables of age, sex, duration of current AAP treatment, HPD-equivalent dosage of current AAP, and 
BMI. Analyses of covariance were used to compare the three treatment groups in terms of the levels of each metabolic component after adjusting for the confounding variables of age, sex, BMI, duration of current AAP treatment, and HPD-equivalent dosage of current AAP.

In the comparison on metabolic components between treatment groups, data are given as real numbers, but log transformation was performed before statistical analyses for data that were not normally distributed. A p-value of $<0.05$ was considered to be statistically significant. We used the SPSS 12.0 version for Windows (IBM, Armonk, NY, USA) for analyses.

Regarding sample size calculation, the event of interest is estimated to occur in 0.25 and 0.574 in the ARP/ZPS group and in the CL/OL/QT group, respectively. The sample size required for an Alpha of 0.05 and $80 \%$ power would be 70 and 28, respectively, 98 in total group, indicating that we may have a change effect with our current results.

\section{RESULTS}

\section{Characteristic of subjects}

Table 1 summarizes the demographic and clinical characteristics of the entire sample $(n=174)$. The mean age was $41.0 \pm 9.9$ years with a mean BMI of $24.8 \pm 4.0 \mathrm{~kg} / \mathrm{m}^{2}$. The mean duration of illness was $16.0 \pm 8.4$ years with a mean duration of currant AAPs of $39.4 \pm 26.7$ months. The duration of illness was much longer than the duration of current AAPs in the study sample.

The antipsychotic-treatment group consisted of the following subgroups: 1) CL/OL/QT ( $\mathrm{n}=61)$; 2) RSP ( $=89$ ); and 3) $\mathrm{ARP} / \mathrm{ZPP}(\mathrm{n}=24)$. In the CL/OL/QT group, CL and OL were used by 34 and 23 subjects, respectively, and four subjects were treated with QT. In the ARP/ZPS group, 20 subjects used ARP, and the remaining four subjects received ZPS.

No significant intergroup differences were observed in age, BMI, outpatient status, use of antidepressants, or severity of psychiatric symptoms as measured by the CGI-S. Selective se-

Table 1. Comparisions of demographic and clinical characteristics of 174 subjects among three antipsychotic groups with different metabolic side effects

\begin{tabular}{|c|c|c|c|c|c|}
\hline & $\mathrm{CL} / \mathrm{OL} / \mathrm{QT}$ & RSP & ARP/ZPS & $F$ & n \\
\hline & $\mathrm{N}=61$ & $\mathrm{~N}=89$ & $\mathrm{~N}=24$ & $\Gamma$ & P \\
\hline Female, N (\%) & $37(60.7)$ & $69(77.5)$ & $14(58.3)$ & $\chi^{2}=6.284$ & 0.043 \\
\hline Outpatients, N (\%) & $48(78.7)$ & $70(78.7)$ & $22(91.7)$ & $\chi^{2}=5.299$ & 0.258 \\
\hline Use of antidepressant, $\mathrm{N}(\%)$ & $13(21.3)$ & $10(11.4)$ & $6(25.0)$ & $\chi^{2}=3.910$ & 0.142 \\
\hline Escitalopram, N (\%) & $7(53.8)$ & $5(50)$ & $3(50)$ & & \\
\hline Sertraline & $2(15.4)$ & $1(10)$ & $1(16.7)$ & & \\
\hline Paroxetine & $2(15.4)$ & $1(10)$ & $1(16.7)$ & & \\
\hline Fluoxetine & & $2(20)$ & & & \\
\hline Trazodone & $1(7.7)$ & $1(10)$ & & & \\
\hline Venlafaxine, N (\%) & $1(7.7)$ & & & & \\
\hline Bupropion, N (\%) & & & $1(16.7)$ & & \\
\hline Age (range) & $40.4 \pm 9.2(19-65)$ & $42.3 \pm 10.2(20-64)$ & $37.3 \pm 9.8(21-58)$ & 2.611 & 0.076 \\
\hline Duration of illness (years) & $17.0 \pm 7.7$ & $16.5 \pm 8.6$ & $11.4 \pm 8.1$ & 4.291 & 0.015 \\
\hline \multicolumn{6}{|l|}{ Previous antipsychotic medication, N (\%) } \\
\hline Drug naïve & $3(5)$ & $8(9)$ & $3(12.5)$ & & \\
\hline RSP & $6(9.8)$ & & $1(4.2)$ & & \\
\hline OL/QT & & $3(3.4)$ & & & \\
\hline $\mathrm{ARP} / \mathrm{HPD} / \mathrm{BPD}$ & $1(1.6)$ & $5(5.6)$ & $1(4.2)$ & & \\
\hline More than two antipsychotics & $7(11.5)$ & $13(14.6)$ & $4(16.0)$ & & \\
\hline Previous psychiatric history before the hospital & $44(72.1)$ & $60(67.4)$ & $15(62.5)$ & & \\
\hline BMI & $25.4 \pm 4.3$ & $24.6 \pm 3.9$ & $23.8 \pm 3.8$ & 1.505 & 0.225 \\
\hline Duration of current AAP, months (range) & $39.5 \pm 26.8(12-127)$ & $43.9 \pm 28.0(12-122)$ & $22.5 \pm 11.1(12-51)$ & 7.177 & 0.001 \\
\hline HPD-equivalent dosage of current AAP (mg/day) & $12.8 \pm 6.1$ & $9.9 \pm 4.0$ & $9.0 \pm 4.5$ & 3.407 & 0.035 \\
\hline CGI-S & $3.4 \pm 0.9$ & $3.5 \pm 0.9$ & $3.1 \pm 0.7$ & 2.658 & 0.073 \\
\hline
\end{tabular}

Real values are given, but log transformation was performed before statistical analyses for data that were not normally distributed (duration of current AAP, HPD-equivalent dosage of current AAP). CL: clozapine, OL: olanzapine, QT: quetiapine, RSP: risperidone, ARP: aripiprazole, ZPS: ziprasidone, BMI: body mass index, AAP: atypical antipsychotic, HPD: haloperidol, CGI-S: Clinical Global Impression-Severity 
rotonin reuptake inhibitors (SSRIs) were the most commonly used antidepressants (84.6\%, 90\% and $83.4 \%$ in three APP group respectively) and escitalopram was the most commonly prescribed antidepressant in all three AAP groups.

The majority of subjects of three AAP group had been treated with another various antipsychotics before entering this study (95\% in CL/OL/QT group, 91\% in RSP group, and 87.5\% in ARP/ZPS group).

Statistically significant differences were observed in sex, duration of illness, duration of current AAP treatment, and HPDequivalent dosage of current AAP. Group RSP included significantly more women compared with group CL/OL/QT or group ARP/ZPS ( $\mathrm{p}=0.043$ ). Patients in the ARP/ZPS group had significantly shorter durations of illness $(\mathrm{p}=0.015)$ and current AAP treatment ( $\mathrm{p}=0.001)$ compared with those in the other two groups. Patients in the CL/OL/QT group received significantly higher HPD-equivalent dosages of their current AAP compared with those in the ARP/ZPR group ( $p=0.035)$. These variables were controlled for in the analyses.

\section{Prevalence of metabolic abnormalities}

Although logistic regression analyses revealed no significant intergroup differences in the prevalence of obesity, the three groups did differ significantly in the prevalence of MetS after adjusting for age, sex, duration of current AAP treatment, and HPD-equivalent dosage of current AAP ( $p=0.001)$. Specifically, MetS was more prevalent in group CL/OL/QT than in group RSP $(p<0.001)$ or group ARP/ZPS ( $p=0.024)$ (Table 2$)$.

The logistic regression analyses also revealed significant differences among groups in terms of the prevalence of hypertriglyceridemia $(p=0.015)$, hyperglycemia $(p=0.022)$, and low HDL-cholesterol ( $\mathrm{p}=0.017$ ) after adjusting for age, sex, BMI, duration of current AAP treatment, and HPD-equivalent dos- age of current AAP.

Hypertriglyceridemia $(\mathrm{p}=0.004)$ and hyperglycemia $(\mathrm{p}=$ 0.006 ) were more prevalent in group CL/OL/QT than in group RSP, respectively. Low HDL-cholesterol was more prevalent in group CL/OL/QT than in group $\mathrm{RSP}(\mathrm{p}=0.043)$ or in group ARP/ ZPS ( $\mathrm{p}=0.008)$.

A non-significant difference in the prevalence of hypertension was observed among groups.

\section{Levels of metabolic parameters}

Triglyceride level $(\mathrm{p}=0.014)$ and the ratio of triglyceride to HDL-cholesterol ( $\mathrm{p}=0.004$ ) were significantly different among three AP groups after adjusting for age, sex, BMI, duration of current AAP treatment, and HPD-equivalent dosage of current AAP (Table 3). Triglyceride levels $(\mathrm{p}=0.004)$ and the ratio of triglyceride to HDL-cholesterol $(\mathrm{p}=0.001)$ was significantly higher in group CL/OL/QT than in group RSP. The ratio of triglyceride to HDL-cholesterol showed a trend-level difference between CL/OL/QT group and ARP/ZPS group ( $\mathrm{p}=0.058$ ).

Fasting plasma glucose level showed a trend-level difference among three AP groups ( $\mathrm{p}=0.052$ ). When stratifying for sex after adjustments were made, we also found a trend-level difference in HDL-cholesterol among female patients $(\mathrm{p}=0.062)$. In female patients, HDL-cholesterol was higher in group ARP/ ZPS than in group CL/OL/QT ( $\mathrm{p}=0.021$ ) or group RSP $(\mathrm{p}=0.037)$. No significant intergroup differences were observed in blood pressure, total cholesterol, or the ratio of total cholesterol to HDLcholesterol.

\section{DISCUSSION}

This naturalistic study revealed that patients with schizophrenia who received different AAPs for 1 year differed signif-

Table 2. Comparisions of the prevalence of metabolic syndrome and its components among three antipsychotic groups with different metabolic side effects

\begin{tabular}{|c|c|c|c|c|c|c|c|}
\hline \multirow{3}{*}{ Metabolic syndrome (\%)* } & \multirow{3}{*}{$\begin{array}{l}\text { CL/OL/QT (1), } \\
\qquad \begin{array}{c}\mathrm{N}=61 \\
57.4\end{array}\end{array}$} & \multirow{3}{*}{$\begin{array}{c}\mathrm{RSP}(2), \\
\mathrm{N}=89 \\
27.0\end{array}$} & \multirow{3}{*}{$\begin{array}{l}\text { ARP/ZPS (3), } \\
\qquad \begin{array}{c}\mathrm{N}=24 \\
25.0\end{array}\end{array}$} & \multirow{3}{*}{$\frac{p}{0.001}$} & \multicolumn{3}{|c|}{ Between groups } \\
\hline & & & & & \multicolumn{2}{|c|}{ Adjusted OR (95\% CI) } & \multirow{2}{*}{$\frac{\mathrm{p}}{<0.001}$} \\
\hline & & & & & 1,2 & $3.990(1.896-8.394)$ & \\
\hline & & & & & 1,3 & $3.624(1.186-11.073)$ & 0.024 \\
\hline Obesity $(\%)^{*}$ & 13.1 & 10.1 & 8.3 & 0.663 & & & \\
\hline Hypertriglyceridemia $(\%)^{\dagger}$ & 52.5 & 23.6 & 25.0 & 0.015 & 1,2 & $3.274(1.462-7.334)$ & 0.004 \\
\hline \multirow[t]{2}{*}{ Low HDL-cholesterolemia $(\%)^{\dagger}$} & 50.8 & 39.3 & 20.8 & 0.017 & 1,2 & $2.205(1.026-4.740)$ & 0.043 \\
\hline & & & & & 1,3 & $5.379(1.549-18.686)$ & 0.008 \\
\hline Hyperglycemia (\%) ${ }^{\dagger}$ & 32.8 & 11.2 & 16.7 & 0.022 & 1,2 & $3.877(1.479-10.166)$ & 0.006 \\
\hline Hypertension $(\%)^{\dagger}$ & 50.8 & 36.0 & 29.2 & 0.615 & & & \\
\hline
\end{tabular}

Obesity, BMI $\geq 30 \mathrm{~kg} / \mathrm{m}^{2}$; Hypertriglyceridemia, fasting plasma triglyceride $\geq 150 \mathrm{mg} / \mathrm{dL}$; low HDL-cholesterolemia, HDL cholesterol $<40 \mathrm{mg} / \mathrm{dL}$ in male, $<50 \mathrm{mg} / \mathrm{dL}$ in female; hyperglycemia, fating plasma glucose $\geq 100 \mathrm{mg} / \mathrm{dL}$; hypertention, systolic $\mathrm{BP} \geq 130 \mathrm{~mm} \mathrm{Hg}$ and/or diastolic BP $\geq 85$ $\mathrm{mm} \mathrm{Hg} .{ }^{*}$ data are adjusted for differences in age, sex, duration of current AAPs, and HPD-equivalent dosage of current AAP, ${ }^{\dagger}$ data are adjusted for differences in age, sex, BMI, duration of current AAP, and HPD-equivalent dosage of current AAP. CL: clozapine, OL: olanzapine, QT: quetiapine, RSP: risperidone, ARP: aripiprazole, ZPS: ziprasidone, BMI: body mass index, HDL: high density lipoprotein, BP: blood pressure, AAP: atypical antipsychotic, HPD: haloperidol 
Table 3. Analyses of covariances of levels of fasting metabolic parameters among three antipsychotic groups with different metabolic side effects

\begin{tabular}{|c|c|c|c|c|c|c|c|}
\hline & $\begin{array}{c}\text { CL/OL/QT (1), } \\
\mathrm{N}=61\end{array}$ & $\begin{array}{c}\mathrm{RSP}(2), \\
\mathrm{N}=89\end{array}$ & $\begin{array}{c}\text { ARP/ZPS (3), } \\
\mathrm{N}=24\end{array}$ & $\mathrm{~F}$ & $\eta p^{2}$ & $\mathrm{p}$ & Post-hoc (p) \\
\hline $\operatorname{BMI}\left(\mathrm{kg} / \mathrm{m}^{2}\right)^{*}$ & $25.4 \pm 4.3$ & $24.6 \pm 3.9$ & $23.8 \pm 3.8$ & 1.143 & 0.013 & 0.321 & \\
\hline Triglyceride $(\mathrm{mg} / \mathrm{dL})^{\dagger}$ & $183.5 \pm 135.1$ & $122.8 \pm 86.0$ & $124.3 \pm 84.6$ & 4.384 & 0.050 & 0.014 & $1>2(0.004)$ \\
\hline HDL-cholesterol $(\mathrm{mg} / \mathrm{dL})^{\dagger}$ & $47.2 \pm 12.1$ & $49.8 \pm 10.1$ & $52.3 \pm 11.1$ & 2.057 & 0.024 & 0.131 & \\
\hline $\operatorname{Male}(\mathrm{mg} / \mathrm{dL})(\mathrm{N})^{\dagger}$ & $46.5 \pm 11.9(24)$ & $48.4 \pm 7.5(20)$ & $48.9 \pm 9.5(10)$ & 0.212 & 0.009 & 0.810 & \\
\hline Female $(\mathrm{mg} / \mathrm{dL})(\mathrm{N})^{\dagger}$ & $47.6 \pm 12.5(37)$ & $50.2 \pm 10.8(69)$ & $54.8 \pm 11.9(14)$ & 2.847 & 0.048 & 0.062 & $1<2(0.021), 2<3(0.037)$ \\
\hline Glucose $(\mathrm{mg} / \mathrm{dL})^{\dagger}$ & $94.8 \pm 24.9$ & $86.8 \pm 16.0$ & $91.8 \pm 38.1$ & 3.009 & 0.035 & 0.052 & $1>2(0.023)$ \\
\hline Total cholesterol $(\mathrm{mg} / \mathrm{dL})^{\dagger}$ & $198.4 \pm 43.8$ & $193.0 \pm 42.0$ & $194.8 \pm 35.4$ & 0.793 & 0.009 & 0.454 & \\
\hline Triglyceride/HDL-cholesterol $^{\dagger}$ & $4.48 \pm 4.48$ & $2.71 \pm 2.23$ & $2.69 \pm 2.37$ & 5.781 & 0.065 & 0.004 & $1>2(0.001), 1>3(0.058)$ \\
\hline Total cholesterol/HDL-cholesterol $^{\dagger}$ & $4.4 \pm 1.4$ & $4.0 \pm 1.1$ & $3.9 \pm 1.1$ & 1.472 & 0.017 & 0.233 & \\
\hline BP, systolic $(\mathrm{mm} \mathrm{Hg})^{\dagger}$ & $123.6 \pm 15.2$ & $121.6 \pm 15.4$ & $119.1 \pm 14.5$ & 0.050 & 0.001 & 0.952 & \\
\hline BP, diastolic $(\mathrm{mm} \mathrm{Hg})^{\dagger}$ & $81.5 \pm 11.0$ & $77.6 \pm 10.2$ & $77.5 \pm 10.0$ & 1.211 & 0.014 & 0.300 & \\
\hline
\end{tabular}

Real values are given, but log transformation was performed before statistical analyses for data that were not normally distributed (Fasting plasma glucose, Triglyceride/HDL-cholesterol). The post-hoc analyses were performed using Bonferroni method. *data are adjusted for differences in age, sex, duration of current AAP and HPD-equivalent dosage of current AAP, ${ }^{\dagger}$ data are adjusted for differences in age, sex, BMI, duration of current AAP, and HPD-equivalent dosage of current AAP. CL: clozapine, OL: olanzapine, QT: quetiapine, RSP: risperidone, ARP: aripiprazole, ZPS: ziprasidone, BMI: body mass index, HDL: high-density lipoprotein, BP: blood pressure, AAP: atypical antipsychotic, HPD: haloperidol

icantly in terms of metabolic disturbances involving lipid and glucose levels even after adjusting for BMI.

The risk of antipsychotic-induced weight gain differs among antipsychotic drugs. ${ }^{3}$ However, we found no significant intergroup differences in the prevalence of obesity or BMI before or after adjusting for clinical variables. We do not consider this finding to contradict the results of previous studies; our results may be attributable to the characteristics of our sample. All subjects had been continuously receiving the same AAP in a naturalistic clinical setting for more than one year. Therefore, those who gained excessive weight may have had their antipsychotics switched to a less metabolically active medication before entering the study. We found that the majority of subjects of three AAP group had been treated with another various antipsychotics before entering this study.

Unlike the prevalence of obesity, the prevalence of MetS differed significantly among the three antipsychotic groups. This finding suggests that obesity is not completely correlated with dysglycemia or dyslipidemia in patients receiving an AAP, which is consistent with the findings reported by Meyer et al. ${ }^{14}$ The high rate of MetS in patients treated with AAPs develop through several mechanisms such as increased appetite, decreased physical activity, weight gain, insulin resistance and direct modification of metabolic parameters. It is important to investigate these potential mechanisms separately. Each potential factors need to be investigated separately to resolve the fundamental mechanism.

Our finding that those in group CL/OL/QT exhibited both more hypertriglyceridemia and higher triglyceride level than those in group RSP is consistent with a previous report that suggested that dibenzodizepine-derived compounds are associated with dyslipidemia. ${ }^{7}$ Individual antipsychotics differ in their tendency to induce hypertriglyceridemia. ${ }^{14-16}$ Several studies have reported positive correlations between changes in triglyceride levels and BMI in dibenzodiazepine-derived compounds. ${ }^{15,17}$ Obesity has been repeatedly associated with insulin resistance, which is believed to be a primary underlying metabolic defect in the development of the characteristic dyslipidemia observed in obesity. ${ }^{18}$ However, our finding of intergroup differences in hypertriglyceridemia after adjusting for BMI is consistent with the findings of several studies of AAPs that found no strong association between the severity of the observed triglyceridemia and weight gain. ${ }^{19-22}$ Therefore, some AAPs may have a direct effect on triglyceride metabolism. Yan et al. ${ }^{23} \mathrm{re}-$ cently reviewed the potential mechanisms by which AAPs may contribute to hypertriglyceridemia and suggested that several lines of evidence indicate that AAPs may have a direct effect on triglyceride metabolism either by stimulating hepatic triglyceride production and secretion or by inhibiting lipoprotein lipase-mediated triglyceride hydrolysis.

A recent study reported the effects of several AAPs (clozapine, risperidone, zipasidone) on lipid metabolism, intracellular cholesterol traffic, and lipid secretion in vitro. ${ }^{24}$ They found that AAPs showed inhibit cholesterol biosynthesis and produce changed in the sterol composition of cells. Assuming that feedback mechanisms are triggered by the decrease in intracellular cholesterol levels and these results are in line with increased expression of gene involved in cholesterol biosynthesis induced 
by AAPs. They also found significant differences among AAPs in terms with the affected enzymes and the extent of activation of enzymes. They suggested that there effects may be relevant in the metabolic side effects of antipsychotics.

Our finding of more prevalent hyperglycemia and higher plasma glucose levels in patients taking CL/OL/QT compared with risperidone after adjusting for BMI is also consistent with the findings of previous reports: patients taking clozapine and olanzapine reportedly exhibit abnormal glucose metabolism, including elevated fasting insulin levels. ${ }^{25,26}$ Additionally, patients receiving risperidone exhibited less severe abnormalities in glucose regulation than did those receiving clozapine or olanzapine. ${ }^{8,25}$ These results support previous findings that clinically significant hyperglycemia can occur during some antipsychotic treatment with or without changes in weight. ${ }^{25}$ Recently, Teff et al. ${ }^{27}$ reported that healthy subjects developed insulin resistance after administration of olanzapine and aripiprazole, along with an absence of weight gain, increase in food intake and hunger, or psychiatric illness, supporting the hypothesis that AAPs exert direct effects on tissue, independent of the mechanisms regulating eating behavior.

Obesity and subjective distress related to weight gain are reportedly predictors of antipsychotic noncompliance. ${ }^{28}$ Because weight gain is easily recognized by patients and clinicians, it is possible to take action to combat obesity. In contrast, it is impossible to detect abnormalities in plasma glucose and lipid levels without laboratory tests. Although many clinical guidelines recommend routine laboratory screening, ${ }^{29,30}$ the rate of metabolic monitoring is concerningly low in people prescribed antipsychotic medication in routine clinical practice. ${ }^{31,32}$

Patients treated with AAPs encounter increased risk of comorbidities. It is well known that the number and the seriousness of comorbid disease which is assessed with comorbidity scoring system such as the Charlson Index ${ }^{33}$ are significantly associated with the mortality rate in longitudinal studies. This fact can be applicable to psychiatric patients with metabolic disturbances. That is, it may be possible that the number and the severity of comorbid metabolic disturbances are likely to have important deleterious effects on mortality rates. Clinicians should make an effort to manage the metabolic side effects.

The strength of the present study is that we controlled for major clinical variables that have significant effects on metabolic status, such as smoking, use of mood stabilizers, and treatment with antidepressants associated with weight gain, by excluding patients who were current smokers and those receiving the aforementioned medications. Smoking is known to be associated with an increased prevalence of MetS independent of BMI class, and this increased risk is related primarily to lower HDL-cholesterol and higher triglyceride levels. ${ }^{34}$ Several mood stabilizers have been associated with weight gain, ${ }^{35}$ and the combination of antipsychotics and mood stabilizers seems to lead to greater weight gain compared with treatment involving one or two mood stabilizers. ${ }^{36}$ Another important strength of this study is that all subjects had been continuously receiving the same antipsychotic medication for more than one year and were treated in a naturalistic clinical setting.

This study has several limitations. First, its cross-sectional design precludes inferences about causal relationships between drug exposure and outcome. Second, the small sample may not be representative of all patients treated with AAPs and is the pivotal drawback to generalize the study finding. Third, even though we controlled for confounding factors which can affect the metabolic states such as smoking and concomitant psychiatric medications, we did not assess other factors that may be related to metabolic disturbances, such as diet and exercise. Subjects were recruited from both inpatient and outpatient settings, which can also affect the metabolic states and activity levels of such patients. Fourth, subjective report on comorbidity is usually known not to be accurate in regarding to MetS related study.

In conclusion, metabolic disturbances are significantly different in AAP groups even after adjusting BMI. AAPs may have direct effect on metabolic parameters. Blood lipid and glucose levels should be monitored regularly regardless of whether patients tend to gain weight.

\section{REFERENCES}

1. Turner MS, Stewart DW. Review of the evidence for the long-term efficacy of atypical antipsychotic agents in the treatment of patients with schizophrenia and related psychoses. J Psychopharmacol 2006;20(6 suppl):20-37.

2. Nasrallah HA. Atypical antipsychotic-induced metabolic side effects: insights from receptor-binding profiles. Mol Psychiatry 2007;13:27-35.

3. Newcomer JW. Second-generation (atypical) antipsychotics and metabolic effects. CNS drugs 2005;19(Suppl 1):1-93.

4. Scigliano G, Ronchetti G. Antipsychotic-induced metabolic and cardiovascular side effects in schizophrenia: a novel mechanistic hypothesis. CNS Drugs 2013;27:249-257.

5. Haupt DW. Differential metabolic effects of antipsychotic treatments. Eur Neuropsychopharmcol 2006;16(Suppl 3):S149-S155.

6. Rummel-Kluge C, Komossa K, Schwarz S, Hunger H, Schmid F, Kissling $\mathrm{W}$, et al. Second-generation antipsychotic drugs and extrapyramidal side effects: a systematic review and meta-analysis of head-to-head comparisons. Schizophrenia Bull 2012;38:167-177.

7. Meyer JM, Koro CE. The effects of antipsychotic therapy on serum lipids: a comprehensive review. Schizophr Res 2004;70:1-17.

8. Henderson DC, Cagliero E, Copeland PM, Borba CP, Evins E, Hayden $\mathrm{D}$, et al. Glucose metabolism in patients with schizophrenia treated with atypical antipsychotic agents: a frequently sampled intravenous glucose tolerance test and minimal model analysis. Arch Gen Psychiatry 2005; 62:19-28.

9. Kang SH, Kim KH, Kang GY, Lee KH, Kim KK, Soh M, et al. Cross-sectional prevalence of metabolic syndrome in Korean patients with schizophrenia. Schizophr Res 2011;128:179-181.

10. De Hert M, Detraux J, van Winkel R, Yu W, Correll CU. Metabolic and cardiovascular adverse effects associated with antipsychotic drugs. Nat Rev Endocrinol 2011;8:114-126.

11. Meyer JM, Davis VG, Goff DC, McEvoy JP, Nasrallah HA, Davis SM, et 
al. Change in metabolic syndrome parameters with antipsychotic treatment in the CATIE Schizophrenia Trial: prospective data from phase 1. Schizophr Res 2008;101:273-286.

12. Grundy SM, Cleeman JI, Daniels SR, Donato KA, Eckel RH, Franklin $\mathrm{BA}$, et al. Diagnosis and management of the metabolic syndrome an American Heart Association/National Heart, Lung, and Blood Institute scientific statement. Circulation 2005;112:2735-2752.

13. World Health Organization. The Asia-Pacific Perspective: Redefining Obesity and Its Treatment. Geneva, Switzerland: World Health Organization; 2000.

14. Meyer JM. A retrospective comparison of weight, lipid and glucose changes between risperidone and olanzapine-treated inpatients: Metabolic outcomes after 1 year. J Clin Psychiatry 2002;63:425-433.

15. Atmaca M, Kuloglu M, Tezcan E, Ustundag B. Serum leptin and triglyceride levels in patients on treatment with atypical antipsychotics. J Clin Psychiatry 2003;64:598-604.

16. Wirshing DA, Boyd JA, Meng LR, Ballon JS, Marder SR, Wirshing WC. The effects of novel antipsychotics on glucose and lipid levels. J Clin Psychiatry 2002;63:856-865.

17. Henderson DC, Cagliero E, Gray C, Nasrallah RA, Hayden DL, Schoenfeld DA, et al. Clozapine, diabetes mellitus, weight gain, and lipid abnormalities: a five-year naturalistic study. Am J Psychiatry 2000;157:975-981.

18. Subramanian S, Chait A. Hypertriglyceridemia secondary to obesity and diabetes. Biochim Biophys Acta 2012;1821:819-825.

19. Markham-Abedi C, de Leon J. Hypertriglyceridemia associated with direct effects of olanzapine rather than with weight gain: a case report. J Clin Psychiatry 2006;67:1473-1474.

20. Meyer JM. Effects of atypical antipsychotics on weight and serum lipid levels. J Clin Psychiatry 2001;62(Suppl 27):27-34; discussion 40-41.

21. Nagamine T. Olanzapine-induced elevation of serum triglyceride levels in a normal weight patient with schizophrenia. Internal Med 2008;47:181182.

22. Reaven GM, Lieberman JA, Sethuraman G, Kraemer H, Davis JM, Blasey C, et al. In search of moderators and mediators of hyperglycemia with atypical antipsychotic treatment. J Psychiatr Res 2009;43:997-1002.

23. Yan H, Chen JD, Zheng XY. Potential mechanisms of atypical antipsychotic-induced hypertriglyceridemia. Psychopharmacology (Berl) 2013; 229:1-7.

24. Canfrán-Duque A, Casado ME, Pastor Ó, Sánchez-Wandelmer J, de la Peña G, Lerma M, et al. Atypical antipsychotics alter cholesterol and fatty acid metabolism in vitro. J Lipid Res 2013;54:310-324.
25. Newcomer JW, Haupt DW, Fucetola R, Melson AK, Schweiger JA, Cooper BP, et al. Abnormalities in glucose regulation during antipsychotic treatment of schizophrenia. Arch Gen Psychiatry 2002;59:337-345.

26. Melkersson KI, Hulting AL, Brismar KE. Elevated levels of insulin, leptin, and blood lipids in olanzapine-treated patients with schizophrenia or related psychoses. J Clin Psychiatry 2000;61:742-749.

27. Teff KL, Rickels MR, Grudziak J, Fuller C, Nguyen HL, Rickels K. Antipsychotic-induced insulin resistance and postprandial hormonal dysregulation independent of weight gain or psychiatric disease. Diabetes 2013; 62:3232-3240.

28. Weiden PJ, Mackell JA, McDonnell DD. Obesity as a risk factor for antipsychotic noncompliance. Schizophr Res 2004;66:51-57.

29. Melkersson K, Dahl ML, Hulting AL. Guidelines for prevention and treatment of adverse effects of antipsychotic drugs on glucose-insulin homeostasis and lipid metabolism. Psychopharmacology (Berl) 2004;175: $1-6$.

30. Tschoner A, Engl J, Laimer M, Kaser S, Rettenbacher M, Fleischhacker WW, et al. Metabolic side effects of antipsychotic medication. Int J Clin Pract 2007;61:1356-1370.

31. Mitchell A, Delaffon V, Vancampfort D, Correll C, De Hert M. Guideline concordant monitoring of metabolic risk in people treated with antipsychotic medication: systematic review and meta-analysis of screening practices. Psychol Med 2012;42:125-147.

32. Barnes TR, Paton C, Cavanagh MR, Hancock E, Taylor DM, et al. A UK audit of screening for the metabolic side effects of antipsychotics in community patients. Schizophrenia Bull 2007;33:1397-1403.

33. Charlson ME, Pompei P, Ales KL, MacKenzie CR. A new method of classifying prognostic comorbidity in longitudinal studies: development and validation. J Chronic Dis 1987;40:373-383.

34. Slagter SN, van Vliet-Ostaptchouk JV, Vonk JM, Boezen HM, Dullaart RP, Kobold AC, et al. Associations between smoking, components of metabolic syndrome and lipoprotein particle size. BMC Med 2013;11:195.

35. Chengappa K, Chalasani L, Brar JS, Parepally H, Houck P, Levine J. Changes in body weight and body mass index among psychiatric patients receiving lithium, valproate, or topiramate: an open-label, nonrandomized chart review. Clin Ther 2002;24:1576-1584.

36. Correll CU. Weight gain and metabolic effects of mood stabilizers and antipsychotics in pediatric bipolar disorder: a systematic review and pooled analysis of short-term trials. J Am Acad Child Adolesc Psychiatry 2007;46:687-700. 